\title{
Recommandations en vue de la prévention des fractures dans les établissements de soins de longue durée
}

\author{
Alexandra Papaioannou MD MSc, Nancy Santesso RD PhD, Suzanne N. Morin MD MSc, Sidney Feldman MD, \\ Jonathan D. Adachi MD, Richard Crilly BSc MD, Lora M. Giangregorio PhD, Susan Jaglal PhD, \\ Robert G. Josse MBBS, Sharon Kaasalainen PhD, Paul Katz MD, Andrea Moser MD MSc, Laura Pickard MA, \\ Hope Weiler RD PhD, Susan Whiting PhD, Carly J. Skidmore MSc, Angela M. Cheung MD PhD; \\ for the Scientific Advisory Council of Osteoporosis Canada
}

Pour la version anglaise, veuillez consulter www.cmaj.ca/lookup/doi/10.1503/cmaj.141331

Balados du JAMC en anglais : Entretien avec l'auteure à l'adresse https://soundcloud.com/cmajpodcasts/141331-guide

$\mathrm{L}$ es Lignes directrices de pratique clinique 2010 pour le diagnostic et le traitement de l'ostéoporose au Canada portaient sur les soins aux adultes vivant dans la communauté. Cependant, le taux de fractures des adultes vivant dans un établissement de soins de longue durée (résidents) est de deux à quatre fois plus élevé que celui des adultes d'âge semblable vivant dans la communauté, et le tiers des aînés qui subissent une fracture de la hanche vivent dans un établissement de soins de longue durée ${ }^{2}$. Les fractures de la hanche sont l'une des conséquences les plus graves de l'ostéoporose et l'une des principales causes d'admission à l'hôpital ${ }^{3}$. Les résidents qui retournent à leur établissement de soins de longue durée après un séjour à l'hôpital ont besoin d'un plus grand nombre d'heures de soins spécialisés ${ }^{4,5}$. De plus, les douleurs associées aux fractures et le délire qu'entraîne souvent l'analgésie sont difficiles à vivre pour les résidents et leur famille. Les fractures vertébrales sont aussi une préoccupation chez les résidents : la prévalence déclarée approche les $30 \%$ (pour au moins une fracture modérée à grave $)^{6}$. Les fractures vertébrales multiples peuvent être une source importante de douleur, d'anxiété, de dépression, de réduction de la fonction pulmonaire ${ }^{7}$ et d'agitation.

Les aînés fragiles qui courent un risque élevé de subir une fracture dans les établissements de soins de longue durée doivent aussi composer avec d'autres problèmes : en effet, plus de $40 \%$ d'entre eux souffrent de démence ${ }^{8}$. une proportion semblable éprouve de la difficulté à avaler $^{9,10}$. et plus de $20 \%$ d'entre eux pourraient souffrir d'insuffisance rénale ${ }^{11,12}$.
Il peut être difficile de dépister les résidents vulnérables aux fractures, étant donné que les outils actuels d'évaluation des risques (l'outil conjoint de l'Association canadienne des radiologistes et d'Ostéoporose Canada ${ }^{13}$ [CAROC; www. osteoporosecanada.ca/multimedia/pdf/CAROC_ FR.pdf] et l'outil d'évaluation du risque de fracture mis au point par l'OMS et adapté au contexte canadien [FRAX; www.shef.ac.uk/FRAX/]) mesurent les risques de fracture sur 10 ans et n'ont pas été validés dans le contexte des soins de longue durée, où plus de $20 \%$ des résidents peuvent décéder dans l'année suivant leur admission ${ }^{14,15}$. Par ailleurs, la plupart des recherches sur l'évaluation des risques et les pharmacothérapies ne portaient pas sur les aînés atteints de multiples comorbidités ${ }^{16,17}$.
Intérêts concurrents : Voir à la fin de l'article.

Cet article a été évalué par des pairs.

Correspondance : Alexandra Papaioannou, papaioannou@hhsc.ca

CMAJ 2015. DOI:10.1503 /cmaj.151124

\section{POINTS CLES}

- Chez les aînés vivant en établissement de soins de longue durée (résidents), les fractures causent de la douleur, de l'agitation, de l'immobilité et des transferts à l'hôpital.

- Les résidents à risque élevé de fracture comprennent ceux qui ont déjà subi une fracture de la hanche ou de la colonne vertébrale, ceux qui ont subi plus d'une fracture et ceux qui ont subi une fracture et qui ont récemment pris des glucocorticoïdes.

- Les recommandations pour la prévention des fractures dans les établissements de soins de longue durée ont été formulées à l'aide de I'approche GRADE (Grading of Recommendations Assessment, Development and Evaluation), qui tient compte de la qualité des données probantes disponibles, de l'équilibre entre les bienfaits et les méfaits, des préférences des résidents et de leurs fournisseurs de soins, ainsi que des ressources nécessaires pour la mise en œuvre de ces recommandations.

- Les stratégies de prévention des fractures, dont les suppléments de vitamine $\mathrm{D}$ et de calcium, I'utilisation de protecteurs de hanche, l'exercice, les interventions multifactorielles pour prévenir les chutes et les traitements pharmacologiques, devraient être adaptées au risque de fracture, à la mobilité, à l'espérance de vie, à la fonction rénale et à la capacité d'avaler de chaque résident. 


\section{Portée}

Le présent document présente des stratégies de prévention des fractures pour les équipes interprofessionnelles prenant soin d'aînés fragiles dans des établissements de soins de longue durée.

\section{Méthodes}

Ces lignes directrices, qui ont été approuvées par Ostéoporose Canada, ont été élaborées au moyen de l'approche GRADE (Grading of Recommendations Assessment, Development and Evaluation) $)^{18,19}$ (www.gradeworkinggroup.org) dans le cadre d'un processus dirigé par une spécialiste de la méthodologie GRADE (N.S.). Le groupe d'experts ayant travaillé sur les lignes directrices était composé des auteurs du présent document, d'autres fournisseurs de soins de santé multidisciplinaires et de chercheurs, ainsi que des représentants de conseils de résidents et de membres de la famille (voir l'annexe 1, accessible au www.cmaj.ca/lookup/ suppl/doi:10.1503/cmaj.141331/-/DC1). On a d'abord demandé au groupe de hiérarchiser une série de questions et de résultats importants. En plus des fractures (de la hanche, vertébrales et non vertébrales), l'ensemble des membres du groupe a considéré comme des résultats importants la douleur, la qualité de vie, la difficulté à accomplir les activités quotidiennes et la perte de mobilité, le décès et les événements indésirables nécessitant des soins médicaux. Pour les membres de la famille des résidents, la prévention de la douleur et le maintien de la mobilité étaient les sujets les plus importants.

Nous avons réalisé des recherches documentaires systématiques pour trouver des méta-analyses en réseaux, des revues systématiques, des essais randomisés contrôlés et des études non randomisées publiés jusqu'en juin 2013. Les détails de la synthèse des données probantes, de la préparation des profils de données probantes et des tableaux portant sur l'application des données probantes à la prise de décision pour cinq grandes stratégies de prévention des fractures (apport en vitamine D et en calcium, médicaments contre l'ostéoporose, protecteurs de hanche, exercice et interventions multifactorielles) sont présentés à l'annexe 1 . Pour résumer, les données probantes sur les risques et les différences relatives de chaque intervention ont été converties en effets absolus accompagnés d'intervalles de confiance (IC) à $95 \%$ et ont été présentées dans les profils de données probantes. Lorsqu'aucune donnée sur les fractures dans le contexte des soins de longue durée n'était disponible, nous avons utilisé les données associées aux adultes de 75 ans et plus. Le taux annuel global de fractures de la hanche, de fractures vertébrales et d'autres fractures chez les résidents d'établissements de soins de longue durée était de $2 \%$ 2,20-22; dans la catégorie de risque élevé, le taux annuel de fractures vertébrales était de $20 \%$, et les taux annuels estimés étaient de $6 \%$ pour les fractures de la hanche et de $6 \%$ pour toutes les autres fractures.

Nous avons évalué la qualité des données probantes comme étant élevée, moyenne, faible ou très faible en fonction des critères GRADE ${ }^{18}$ (tableau 1). Les tableaux portant sur l'application des données probantes à la prise de décision soumis à l'examen du groupe d'experts comportaient un résumé des données probantes relatives aux bienfaits et aux effets néfastes, une évaluation de la qualité des données probantes et des renseignements sur les valeurs et les préférences d'intérêt des résidents et de leur famille, l'utilisation des ressources et la faisabilité. Les recommandations ont été classées comme fortes ou conditionnelles (tableau 2). Les fortes recommandations commencent par la formule «nous recommandons », tandis que les recommandations conditionnelles commencent par la formule «nous suggérons ».

\section{Recommandations}

Nous avons formulé des recommandations d'interventions visant la prévention des fractures dans deux groupes, soit les résidents âgés d'établissements de soins de longue durée qui courent un risque élevé de fracture et les résidents âgés qui ne sont pas particulièrement vulnérables aux fractures.

Tableau 1 : Qualité des données probantes : confiance dans l'effet ${ }^{18}$

Classement de la qualité des données

Élevée

Moyenne

Faible

Très faible

\section{Définition}

Nous avons pleinement confiance que l'effet réel est proche de l'estimation.

Nous avons une confiance moyenne dans l'estimation de l'effet. L'effet réel est probablement proche de l'estimation, mais il pourrait aussi être très différent.

Notre confiance dans l'estimation de l'effet est limitée. L'effet réel pourrait être très différent de l'estimation.

Nous avons très peu confiance en l'estimation de l'effet. L'effet réel est probablement très différent de l'estimation. 
Comme aucune méthode permettant de définir les personnes à risque élevé de fracture dans les établissements de soins de longue durée n'a été validée pour le moment, nous proposons une adaptation de la définition présentée dans les Lignes directrices de pratique clinique 2010 pour le diagnostic et le traitement de l'ostéoporose au Canada ${ }^{1}$. qui repose grandement sur les antécédents de fracture (encadré 1). Si on considère qu'un résident court un risque élevé de fracture et qu'il a reçu des traitements contre l'ostéoporose avant d'être admis dans un établissement de soins de longue durée, cette classification pourrait continuer de s'appliquer au moment de l'admission.

Le dépistage de fractures vertébrales décrit dans les lignes directrices de $2010^{1}$ est recommandé. Si on effectue une radiographie de profil en même temps qu'une radiographie pulmonaire, il est possible de demander du même coup de rechercher des fractures vertébrales.

\section{Calcium et vitamine D}

\section{Calcium}

Pour tous les résidents, nous recommandons des interventions alimentaires afin que l'apport nutritionnel recommandé en calcium soit respecté (forte recommandation; données probantes de qualité moyenne).

Cette recommandation accorde une grande valeur à la diminution des fractures, de la mortalité et des chutes et une valeur moindre aux ressources nécessaires dans les établissements de soins de longue durée pour mettre en place les interventions visant à assurer un apport alimentaire adéquat en calcium. Elle est fondée sur les résultats d'une évaluation des effets des suppléments de calcium, qui ont été utilisés comme preuves directes de l'apport alimentaire; cependant, les interventions alimentaires n'ont pas les effets néfastes des suppléments. Pour les personnes de plus de 70 ans, l'apport nutritionnel recommandé en calcium est de $1200 \mathrm{mg}$ par jour (trois portions de produits laitiers ou de substituts).

Pour les résidents à risque élevé de fracture qui sont incapables d'atteindre l'apport nutritionnel recommandé en calcium par l'alimentation, nous recommandons la prise quotidienne de $500 \mathrm{mg}$ de suppléments de calcium (forte recommandation; données probantes de qualité moyenne).

Pour les résidents qui ne sont pas à risque élevé de fracture qui sont incapables d'atteindre l'apport nutritionnel recommandé en calcium par l'alimentation, nous suggérons la prise quotidienne d'une quantité maximale de $500 \mathrm{mg}$ de suppléments de calcium, selon les ressources disponibles et les valeurs et préférences des résidents ou de leurs soignants (recommandation conditionnelle; données probantes de qualité moyenne).

La recommandation visant les résidents à risque élevé de fracture accorde une grande valeur à la diminution des fractures de la hanche et à la légère diminution des fractures vertébrales et non vertébrales et de la mortalité qui peuvent être rendues possibles par la supplémentation en calcium. Elle accorde une valeur moindre au léger risque accru de symptômes gastro-intestinaux indésirables et aux ressources nécessaires dans un établissement de soins de longue durée pour offrir une supplémentation en calcium.

La recommandation visant les résidents qui ne sont pas à risque élevé est conditionnelle, car il est possible que la supplémentation en calcium ne procure que peu de bienfaits, voire aucun, tout en entraînant des effets indésirables comme des symptômes gastro-intestinaux et rénaux. Pour les résidents qui tiennent à éviter ces effets indésirables, la supplémentation pourrait ne pas être souhaitable.

Ces recommandations s'appliquent à la supplémentation avec tout composé de calcium, y compris le carbonate de calcium et le citrate de calcium. La recommandation de limiter la supplémentation à $500 \mathrm{mg}$ est fondée sur l'incertitude quant aux effets néfastes de la supplémentation en calcium dans les

Tableau 2 : Interprétation des recommandations fortes et conditionnelles pour la prévention des fractures ${ }^{19}$

Type de recommandation; signification pour le groupe cible

Groupe cible

Patients

Cliniciens
Forte recommandation (« Nous recommandons... »)
Recommandation conditionnelle (" Nous suggérons... »)
La plupart des personnes dans cette situation souhaiteraient suivre les mesures recommandées, et seules quelques-unes ne le voudraient pas.

La plupart des personnes devraient recevoir l'intervention.
La majorité des personnes dans cette situation souhaiteraient suivre les mesures suggérées, mais beaucoup ne le voudraient pas.

Les cliniciens reconnaissent que différents choix seront appropriés selon les patients et qu'ils doivent aider chaque personne à prendre une décision de gestion cohérente avec ses valeurs et ses préférences. 
études auprès de personnes vivant dans la communauté ayant reçu une supplémentation en calcium d'au moins 1000 mg par jour. Par ailleurs, les bienfaits de la supplémentation en calcium sont étroitement liés à un apport adéquat en vitamine $\mathrm{D}$.

\section{Vitamine D}

Pour les résidents à risque élevé de fracture, nous recommandons la prise quotidienne de suppléments de 800 à 2000 UI de vitamine $D_{3}$ (forte recommandation; données probantes de qualité moyenne).

Pour les résidents qui ne courent pas un risque élevé de fracture, nous suggérons la supplémentation quotidienne de 800 à 2000 UI de vitamine $D_{3}$ afin d'atteindre l'apport nutritionnel recommandé, selon les ressources disponibles et les valeurs et préférences des résidents ou de leurs soignants (recommandation conditionnelle; données probantes de qualité moyenne).

La recommandation visant les résidents à risque élevé accorde une grande valeur à la diminution des fractures de la hanche, de la mortalité et des chutes, et une valeur moindre aux ressources nécessaires dans un établissement de soins de longue durée pour offrir une supplémentation en vitamine D. La recommandation visant les résidents qui ne courent pas un risque élevé accorde aussi une grande valeur à la diminution des chutes, étant donné qu'elles peuvent entraîner des blessures graves et de la peur et qu'elles constituent un fardeau important pour le personnel des soins de longue durée. Toutefois, une certaine incertitude demeure en ce qui concerne la diminution du nombre de chutes et la diminution faible, voire inexistante des fractures avec la supplémentation en vitamine $\mathrm{D}$ dans ce groupe.

Ces recommandations s'appliquent à la supplémentation en vitamine $\mathrm{D}_{3}$, une forme qui pourrait être plus facilement accessible en raison de son coût moindre par comparaison à la vitamine $\mathrm{D}_{2}$. Une dose d'environ 800 UI a entraîné une diminution des fractures chez les personnes dont le taux de 25-hydroxyvitamine D était normal ou faible ${ }^{24}$ ainsi que la normalisation du taux de 25-hydroxyvitamine D lorsque ce taux était faible ${ }^{25}$; par conséquent, une quantité de 800 UI est recommandée. Cependant, la dose exacte

\section{Encadré 1 : Facteurs indiquant un risque élevé de fracture*}

Résidents d'établissements de soins de longue durée présentant un des facteurs suivants :

- fracture antérieure de la hanche ou de la colonne vertébrale;

- plus d'une fracture antérieuret;

- prise récente de glucocorticoïdes systémiques et une fracture antérieuret;

- reconnu à risque élevé ou traité pour l'ostéoporose avant l'arrivée à l'établissement de soins de longue durée.

* Adapté des lignes directrices 2010 d'Ostéoporose Canada'.

† À l'exception des fractures des mains, des pieds ou des chevilles. peut dépendre de la posologie disponible (p. ex., une goutte ou un comprimé de 1000 UI serait acceptable). Les bienfaits de la supplémentation en vitamine $\mathrm{D}$ sont étroitement liés à un apport adéquat en calcium; les recommandations concernant l'apport en calcium devraient donc aussi être appliquées. L'apport nutritionnel recommandé en vitamine $\mathrm{D}$ chez les personnes de plus de 70 ans est de 800 UI quotidiennement, et l'apport maximal tolérable est de 4000 UI.

\section{Résumé des données probantes}

Dans l'ensemble, en ce qui concerne le calcium et la vitamine $\mathrm{D}$, des données probantes de qualité moyenne montrent des bienfaits, et des données probantes de qualité faible à très faible montrent des méfaits. Nous avons conclu que la vitamine D conjuguée au calcium réduit probablement les fractures de la hanche et la mortalité davantage que la vitamine $\mathrm{D}$ ou le calcium pris séparément $^{24,26,27}$ : chez les résidents à risque élevé, nous avons estimé que cela correspond à une diminution de 15 fractures de la hanche (IC à $95 \%:-24$ à -5) pour 1000 sujets; chez les résidents qui ne sont pas à risque élevé, nous avons estimé la diminution à 5 fractures de la hanche (IC à $95 \%$ : -8 à -2) pour 1000 sujets; et chez tous les résidents, nous avons estimé qu'il survenait 7 décès de moins (IC à $95 \%$ : -14 à -1) pour 1000 sujets.

Nous avons constaté que la supplémentation en vitamine $\mathrm{D}$ et en calcium a probablement peu ou pas d'effet sur les fractures vertébrales : seulement 2 fractures vertébrales de moins (IC à $95 \%$ : -44 à 61) ont été constatées pour 1000 sujets. L'effet est semblable avec la vitamine $\mathrm{D}$ prise seule, mais une diminution serait probable avec le calcium seulement (49 fractures de moins [IC à $95 \%$ : -99 à 19] pour 1000 sujets) ${ }^{26,27}$. Le calcium, ou la vitamine D conjuguée ou non au calcium, a probablement peu ou pas d'effet sur l'incidence des fractures non vertébrales ${ }^{24,26,27}$, la qualité de $v^{28}{ }^{28}$ ou la force musculaire ${ }^{29}$.

Les données sur les chutes n'étaient pas précises (les IC englobant à la fois les possibilités de bienfaits, d'effets nuls et de méfaits), et les effets n'étaient pas constants lorsque le taux ou le risque de chutes était mesuré ${ }^{30-33}$. Cependant, la vitamine $\mathrm{D}$ et le calcium, ou la vitamine D seule, pourraient entraîner une diminution des chutes. Ceci est important, car le tiers des chutes peut entraîner des blessures, et une chute sur cinq causant des blessures peut contraindre le patient à recevoir des soins hors de son milieu habituel ${ }^{34}$. Nous n'avons trouvé aucune donnée sur la douleur, l'anxiété, la mobilité et l'accomplissement des activités quotidiennes relativement au calcium et à la vitamine $\mathrm{D}$. 
En ce qui concerne les événements indésirables mineurs et majeurs, les suppléments de vitamine D ou de calcium augmentent probablement les symptômes gastro-intestinaux faibles ou importants dans une mesure semblable, à raison d'environ 8 incidents (IC à $95 \%: 0$ à 17 ) pour 1000 sujets $^{26}$. Les symptômes gastro-intestinaux ou les difficultés à prendre des comprimés de calcium pourraient contribuer à la faible adhésion au traitement ${ }^{28,33}$. Les données probantes indiquent une faible hausse des cas d'hypercalcémie ( 5 cas de plus pour 1000 sujets, IC à $95 \%$ : -1 à 18) et d'insuffisance rénale ou de calculs rénaux ( 3 cas de plus pour 1000 sujets, IC à $95 \%$ : 0 à 6) avec la prise de vitamine $\mathrm{D}\left(\mathrm{D}_{2} \text { ou } \mathrm{D}_{3}\right)^{26}$. La présence d'une augmentation du nombre d'infarctus du myocarde avec la prise de supplémentation en calcium de $1000 \mathrm{mg}$ ou plus chez les personnes vivant dans la communauté est incertaine, puisqu'une telle augmentation ne correspond pas aux diminutions observées de la mortalitéé ${ }^{26}$,et que les IC associés aux estimations englobent l'absence d'effet et la possibilité de méfaits importants ${ }^{35-37}$.

Les analyses par sous-groupes issues de revues systématiques ont montré qu'il pourrait y avoir peu ou pas de différence dans les taux de fractures ou de chutes selon le type de vitamine $\mathrm{D}\left(\mathrm{D}_{3} \text { ou } \mathrm{D}_{2}\right)^{26,30,38}$, que les bienfaits pourraient être plus importants avec une quantité de vitamine D supérieure à 792 UI (l'apport réel dans la plupart des études se chiffrait entre 792 et 844 UI), mais aucune différence n'a été constatée avec moins ou plus de $1000 \mathrm{mg}$ de calcium; en outre, lorsque la vitamine $\mathrm{D}$ est donnée en fortes doses mensuelles ou annuelles, les effets ne sont pas constants ${ }^{24,39}$. Les analyses ont bien montré que la vitamine $\mathrm{D}$ pourrait contribuer davantage à la réduction des chutes ${ }^{30,32}$ et des fractures chez les personnes dont le taux de vitamine D est faible ${ }^{24}$. Autier et ses collaborateurs ${ }^{25}$ ont aussi constaté que la prise d'environ 800 UI quotidiennement pendant plusieurs mois peut normaliser le taux sérique de vitamine $\mathrm{D}$ chez les personnes ayant initialement une déficience en vitamine D (p. ex., $\leq 25 \mathrm{nmol} / \mathrm{l}$ ).

\section{Traitement pharmacologique pour les personnes à risque élevé de fracture}

En formulant nos recommandations concernant le traitement pharmacologique pour les personnes à risque élevé de fracture, nous avons accordé une grande valeur aux problèmes fréquents chez les aînés qui pourraient compromettre l'administration sécuritaire de ces médicaments, ainsi qu'aux risques immédiats de fractures et aux conséquences des chutes et des fractures, notamment l'augmentation de la douleur, la perte de mobilité et le risque accru de décès.
Nous avons évalué les effets des bienfaits et des méfaits à partir d'un an; ainsi, ces recommandations s'appliquent à des aînés dont l'espérance de vie est supérieure à un an.

Pour les résidents à risque élevé de fracture, nous recommandons l'alendronate (prise hebdomadaire) ou le risedronate (prise hebdomadaire ou mensuelle) comme traitement de première intention (forte recommandation; données probantes de qualité moyenne).

Les diminutions probables du risque de fractures (de la hanche, vertébrales et non vertébrales) et de la mortalité associées à la prise d'alendronate ou de risedronate l'emportent sur les risques faibles ou incertains de méfaits ou d'effets indésirables, comme des fractures atypiques du fémur. L'alendronate et le risedronate sont recommandés comme traitements de première intention en raison de leur coût peu élevé par comparaison à d'autres traitements. Il ne faut pas écraser les comprimés d'alendronate et de risedronate. Par ailleurs, ces médicaments doivent être utilisés seulement avec des aînés qui peuvent rester assis pendant 30 minutes après l'administration. Certaines formulations doivent être administrées au moins 30 minutes avant de manger, tandis que d'autres peuvent être prises avec des aliments.

En ce qui concerne les aînés qui n'arrivent pas à avaler ou qui ont de la difficulté à prendre des médicaments par voie orale, d'autres traitements de première intention peuvent être envisagés (voir les recommandations ci-dessous concernant le denosumab et l'acide zolédronique). Les monographies de produit indiquent que l'alendronate ${ }^{40}$ et le risedronate ${ }^{41}$ ne sont pas recommandés pour les aînés souffrant d'insuffisance rénale grave (clairance de la créatinine $<35 \mathrm{ml} / \mathrm{min}$ ou $<30 \mathrm{ml} / \mathrm{min}$, respectivement).

Pour les résidents à risque élevé de fracture qui ont de la difficulté à prendre des médicaments par voie orale, nous recommandons l'acide zolédronique comme traitement de première intention (forte recommandation; données probantes de qualité moyenne).

Les diminutions probables du risque de fractures (de la hanche, vertébrales et non vertébrales) et de la mortalité entraînées par la prise d'acide zolédronique l'emportent légèrement sur les risques accrus incertains d'effets indésirables sur le plan musculosquelettique (arthralgie, myalgie) et le coût plus élevé par comparaison à d'autres traitements de première intention. Cette recommandation s'applique aux aînés qui ont de la difficulté à prendre des médicaments par voie orale pour cause de dysphagie, qui sont incapables de rester 
assis pendant 30 minutes, qui souffrent de troubles cognitifs ou qui ont une intolérance aux autres médicaments. Selon la monographie de produit de l'acide zolédronique ${ }^{42}$, la perfusion doit durer au moins 15 minutes. Par ailleurs, Santé Canada recommande de faire preuve de prudence avec les personnes prenant des médicaments susceptibles de perturber la fonction rénale, de surveiller la clairance de la créatinine avant le traitement et régulièrement après le traitement, d'assurer l'hydratation des patients $(500 \mathrm{ml} \mathrm{d}$ 'eau $)$ avant et après le traitement et d'éviter d'administrer ce médicament à des personnes dont la fonction rénale est gravement atteinte (clairance de la créatinine $<30 \mathrm{ml} / \mathrm{min})^{43}$.

Pour les résidents à risque élevé de fracture qui ont de la difficulté à prendre des médicaments par voie orale, nous recommandons le denosumab comme traitement de première intention (forte recommandation; données probantes de qualité moyenne).

Les diminutions du risque de fractures (de la hanche, vertébrales et non vertébrales) et de la mortalité entraînées par la prise de denosumab l'emportent légèrement sur les risques faibles et incertains d'infections graves et le coût plus élevé par comparaison à d'autres traitements de première intention. Cette recommandation s'applique aux aînés qui ont de la difficulté à prendre des médicaments par voie orale pour cause de dysphagie, qui sont incapables de rester assis pendant 30 minutes, qui souffrent de troubles cognitifs ou qui ont une intolérance aux autres médicaments. Bien que le denosumab puisse être prescrit aux résidents dont la fonction rénale est atteinte, la monographie de ce médicament ${ }^{44}$ indique que dans le cadre d'études cliniques, les patients dont la fonction rénale était atteinte (clairance de la créatinine $<30 \mathrm{ml} / \mathrm{min}$ ) et les patients sous dialyse couraient un plus grand risque d'hypocalcémie que ceux dont la fonction rénale était normale.

Pour les résidents à risque élevé de fracture, nous suggérons la tériparatide (recommandation conditionnelle; données probantes de qualité moyenne).

Bien que les bienfaits de la tériparatide (particulièrement dans le cas de fractures vertébrales) l'emportent probablement sur les méfaits possibles du traitement, le coût du traitement en restreint l'accès, et il se pourrait que le fardeau associé soit plus élevé en raison des injections quotidiennes. Une faible valeur a été accordée à l'effet incertain de la tériparatide sur la dorsalgie en raison de fractures vertébrales passées et à venir, ainsi qu'aux fractures de la hanche.
Pour les résidents à risque élevé de fracture, nous suggérons de ne pas utiliser le raloxifène (recommandation conditionnelle; données probantes de qualité moyenne).

Les méfaits du raloxifène (notamment la thromboembolie veineuse et les problèmes musculosquelettiques, comme l'arthralgie et la myalgie) l'emportent probablement sur la diminution probable de fractures vertébrales et les légères diminutions des fractures non vertébrales et de la hanche.

Pour les résidents à risque élevé de fracture, nous suggérons de ne pas utiliser l'étidronate (recommandation conditionnelle; données probantes de qualité moyenne).

Des données probantes de qualité moyenne indiquent peu ou pas de diminution des fractures (particulièrement dans le cas des fractures de la hanche) avec la prise d'étidronate. Par ailleurs, compte tenu de l'absence de bienfaits importants, le coût de ce médicament est élevé.

\section{Résumé des données probantes}

Des données probantes de qualité moyenne à l'appui de pharmacothérapies ont été obtenues à partir de méta-analyses en réseaux faites sur des essais randomisés contrôlés menés auprès de plus de 100000 personnes à risque élevé de fracture $^{27,38,45}$. Certaines études comportaient un risque de partialité et de l'incertitude lorsque les effets chez les femmes postménopausées étaient appliqués aux résidents d'établissements de soins de longue durée. Les résultats indiquent des diminutions probables d'environ 25 fractures de la hanche pour 1000 sujets avec tous les médicaments, mais des diminutions relativement moindres avec la prise d'étidronate et de raloxifène. Les études ont aussi montré une diminution probable des fractures vertébrales (environ 100 fractures pour 1000 sujets) et des fractures non vertébrales (environ 20 pour 1000 sujets) pour tous les médicaments, mais une diminution plus marquée avec la tériparatide et une diminution moins marquée avec le raloxifène ont été constatées. Des revues systématiques ont conclu que des diminutions des taux de mortalité étaient possibles avec les bisphosphonates (10 de moins [IC à $95 \%$ : -22 à 3] pour 1000 sujets), le raloxifène (10 de moins [IC à $95 \%:-21$ à 0 ] pour 1000 sujets) et le denosumab ( 23 de moins [IC à $95 \%$ : -46 à 6] pour 1000 sujets $)^{46}$. D'après des données probantes de faible qualité, d'autres bienfaits pourraient être associés à ces traitements, comme une légère diminution de la dorsalgie relativement à des fractures vertébrales passées et à venir pour la tériparatide ${ }^{47}$, mais un effet faible, voire inexistant a été constaté quant à la qualité de vie avec d'autres traitements ${ }^{48-51}$. 
Des données probantes de qualité faible à très faible indiquent de très légers risques de problèmes graves comme l'ostéonécrose de la mâchoire et les fractures atypiques ou la guérison lente (estimations à $<1$ pour 10000 aînés vivant dans la communauté $)^{52-54}$. Nous n'avons pu déterminer si ces risques sont plus élevés chez les résidents d'établissements de soins de longue durée. Les données probantes indiquant un risque de fibrillation auriculaire avec les bisphosphonates ${ }^{55}$ et de problèmes cérébrovasculaires ou cardio-vasculaires avec le raloxifène étaient aussi de faible qualités6; ces résultats étaient imprécis et englobaient la possibilité d'augmentations faibles ou inexistantes de ces problèmes. Le risque de thromboembolie veineuse pourrait augmenter avec la prise de raloxifène (12 cas de plus [IC à $95 \%$ : 7 à 19] pour 1000 sujets $)^{56}$, le risque de problèmes musculosquelettiques pourrait augmenter avec la prise d'acide zolédronique (146 cas de plus [IC à $95 \%$ : 125 à 169] pour

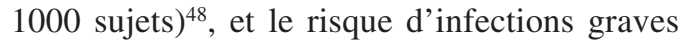
pourrait augmenter avec la prise de denosumab (8 cas de plus [IC à $95 \%$ : 0 à 18] pour

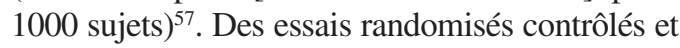
la pharmacovigilance relativement aux bisphosphonates et au raloxifène ont montré peu ou pas d'effet par ces médicaments sur les symptômes gastro-intestinaux graves ${ }^{38}$.

Nous estimons que les coûts directs des médicaments sont justifiés compte tenu des bienfaits globaux qu'apportent la plupart d'entre eux, à l'exception de l'étidronate et du raloxifène. Cependant, les coûts de l'acide zolédronique, du denosumab et de la tériparatide sont élevés par comparaison à d'autres traitements.

\section{Protecteurs de hanche}

Pour les résidents mobiles et à risque élevé de fracture, nous recommandons des protecteurs de hanche (forte recommandation; données probantes de qualité moyenne).

Pour les résidents mobiles qui ne sont pas à risque élevé de fracture, nous suggérons des protecteurs de hanche, selon les ressources disponibles et les valeurs et préférences des résidents (recommandation conditionnelle; données probantes de qualité moyenne).

Ces recommandations accordent une grande valeur au fait d'éviter les conséquences graves des fractures de la hanche chez les résidents mobiles, y compris la douleur, la perte de mobilité et le décès. Une valeur moindre a été accordée au coût ou au fardeau pour la personne ou les ressources de l'établissement de soins de longue durée. Étant donné la faible réduction des fractures de la hanche obtenue à l'aide des protecteurs, les aînés qui ne sont pas à risque élevé peuvent choisir d'autres options de prévention des fractures. Il est reconnu qu'il peut être difficile de suivre la recommandation de porter des protecteurs de hanche; des stratégies pour améliorer l'adhésion pourraient s'avérer nécessaires. Cette recommandation s'applique aux protecteurs durs et mous, et le choix entre ces options dépend des préférences personnelles.

\section{Résumé des données probantes}

Des données probantes de qualité moyenne provenant de revues systématiques montrent une réduction relative du risque de fractures de la hanche de $18 \%$ (IC à $95 \%$ : $0 \%$ à $33 \%$ ) chez les aînés portant des protecteurs de hanche en établissement de soins de longue durée ${ }^{58}$. Durant une année, une réduction de quatre fractures de la hanche (IC à $95 \%$ : -7 à 0) pour 1000 aînés portant des protecteurs serait possible, de même qu'une réduction de 11 fractures chez les aînés à risque élevé (IC à $95 \%$ : -20 à 0). Toutefois, une fracture du bassin de plus (IC à $95 \%$ : 0-4) pour 1000 aînés qui ne sont pas à risque élevé, et huit de plus (IC à $95 \%$ : -3 à 30) pour 1000 aînés qui le sont pourrait être probable. Des données probantes de qualité moyenne montrent aussi que la différence dans la fréquence des chutes ou des événements indésirables nécessitant une intervention médicale est probablement faible ou nulle, et que les événements indésirables mineurs, comme l'irritation de la peau, survenaient chez moins de $2 \%$ des personnes portant des protecteurs de hanche (durs ou mous). L'effet sur la qualité de vie et la mortalité est incertain, et les données sur la douleur, l'anxiété, la mobilité et l'accomplissement des activités quotidiennes n'étaient pas disponibles. L'adhésion à l'utilisation de protecteurs de hanche variait selon les études de $24 \%$ à $80 \%$. L'incidence de l'adhésion sur les effets des protecteurs est incertaine, mais les effets observés pourraient représenter les effets réels observés lors de la mise en œuvre de cette stratégie.

\section{Exercice}

Pour les résidents qui ne sont pas à risque élevé de fracture, nous suggérons des exercices d'équilibre, de force et d'entrainement fonctionnel pour prévenir les chutes (recommandation conditionnelle; données probantes de qualité moyenne).

Cette recommandation accorde une grande valeur à la faible réduction des chutes qui est probablement obtenue par l'exercice, puisque les chutes peuvent entraîner des blessures graves. Elle accorde aussi une grande valeur aux autres avantages offerts par l'exercice et une valeur moindre aux coûts incertains de la mise en place de programmes d'exercice dans les établissements de soins de longue durée. 
Pour les résidents à risque élevé de fracture, nous ne suggérons des exercices d'équilibre, de force et d'entrainement fonctionnel que s'ils font partie d'une intervention multifactorielle pour prévenir les chutes (recommandation conditionnelle; données probantes de faible qualité).

Cette recommandation accorde une grande valeur au fait d'éviter la faible augmentation des chutes qui peut survenir chez les personnes à risque élevé de chute participant à des exercices d'équilibre, de force et d'entraînement fonctionnel. Certains aînés peuvent tenir à l'exercice malgré le risque potentiel de chutes. Lorsque des exercices sont offerts aux résidents, ils devraient faire partie d'une intervention multifactorielle pour prévenir les chutes (comprenant une analyse des médicaments [p. ex., à l'aide des critères de Beers $\left.{ }^{59}\right]$, une évaluation des dangers environnementaux ou l'utilisation d'appareils d'assistance) ou être offerts avec d'autres interventions pour prévenir les fractures (y compris les suppléments de vitamine $\mathrm{D}$ et de calcium, les protecteurs de hanche et les traitements pharmacologiques).

\section{Résumé des données probantes}

Ces recommandations sont fondées sur des données ayant fait l'objet d'une revue systématique de qualité moyenne à faible ${ }^{31}$. Cette revue comprenait des analyses par sous-groupe d'aînés dans des établissements de soins de haut niveau et de niveau intermédiaire, qui ont servi à orienter les recommandations pour les personnes à risque élevé ou non de fractures, respectivement. La plupart des études ne mesuraient pas le nombre de fractures, la qualité de vie, la mobilité ou la douleur. C'était plutôt le risque de chutes qui était utilisé pour orienter la recommandation. Les coûts n'ont pas été examinés.

Les analyses par sous-groupe des soins de haut niveau et de niveau intermédiaire chez les résidents âgés à risque élevé de fracture semblaient indiquer des augmentations du nombre de chutes (870 de plus [IC à $95 \%$ : -210 à 2370] pour 1000 sujets) et du nombre d'aînés qui subissent une chute ( 85 de plus [IC à $95 \%$ : -20 à 210] pour 1000 sujets). Chez les aînés qui ne sont pas à risque élevé de fracture, les analyses pointaient vers des réductions du nombre de chutes (660 de moins [IC à $95 \%$ : -1290 à 390] pour 1000 sujets) et du nombre d'aînés qui subissent une chute (20 de moins [IC à $95 \%$ : -115 à 105] pour 1000 sujets). Ces résultats proviennent d'études qui évaluaient des exercices d'équilibre (comme le tai-chi), de force et d'entraînement fonctionnel. Une étude a mesuré l'incidence de fractures de la hanche, mais les résultats étaient incertains en raison du petit nombre d'incidents. Une revue systématique de l'exercice dans le cadre d'une intervention multi- factorielle pour prévenir les chutes a montré que l'intervention multifactorielle pourrait réduire les chutes et le nombre de fractures de la hanche ${ }^{31}$.

\section{Interventions multifactorielles}

Pour tous les résidents, nous suggérons des interventions multifactorielles conçues sur mesure pour réduire le risque de chutes et de fractures (recommandation conditionnelle; données probantes de faible qualité).

Les interventions multifactorielles sont toutes les combinaisons d'interventions destinées à réduire les chutes conçues en fonction du risque individuel. Ces interventions peuvent comprendre des revues des médicaments (p. ex., à l'aide des critères de Beers ${ }^{59}$ ), l'évaluation des dangers environnementaux, l'utilisation d'appareils d'assistance, l'exercice, la gestion de l'incontinence urinaire et les interventions éducatives pour le personnel. Cette recommandation est conditionnelle, en raison de la faible qualité des données probantes concernant les bienfaits importants mais légers, et les coûts inconnus et potentiellement élevés de la mise en œuvre d'interventions multifactorielles dans les établissements de soins de longue durée. Une grande valeur a été accordée aux petites réductions des chutes qui peuvent se produire, puisque celles-ci peuvent entrainer des blessures graves. Nous n'avons pas suggéré d'interventions particulières pour les interventions multifactorielles, puisqu'il n'a pas été possible de déterminer quelle combinaison de stratégies offre des bienfaits. Il sera important de prendre en compte le niveau de risque de fracture du résident et d'adapter les stratégies en conséquence.

\section{Résumé des données probantes}

Les données tirées d'une revue systématique des interventions destinées à prévenir les chutes chez les aînés dans les établissements de soins étaient de faible qualité en raison du risque de partialité des études analysées et du degré moyen à élevé d'incohérence des effets d'une étude à l'autre, qui ne pouvait pas être expliqué par le niveau de soins, la cognition ou la combinaison d'interventions ${ }^{31}$. La plupart des études ne mesuraient pas le nombre de fractures, la qualité de vie, la mobilité ou la douleur; le risque de chutes a donc été utilisé pour orienter cette recommandation.

Dans l'ensemble, la revue systématique semblait indiquer des réductions du nombre de chutes (660 de moins [IC à $95 \%:-1230$ à 120] pour 1000 sujets par année) et du nombre de résidents ayant subi une chute (55 de moins [IC à $95 \%$ : -115 à 10] pour 1000 sujets) avec l'utilisation d'interventions multifactorielles. Des données probantes de faible qualité indiquaient une réduc- 


\section{Évaluation du risque de fracture à} l'arrivée

- Fracture antérieure de la hanche?

- Fracture vertébrale antérieure?

- Plus d'une fracture antérieure (à I'exception des mains, des pieds et des chevilles)?

- Prise récente de glucocorticoïdes et une fracture antérieure (à l'exception des mains, des pieds et des chevilles)?

- Évalué à risque élevé de fracture et traité pour une fracture avant l'arrivée?

- Fracture vertébrale présente? (si une radiographie pulmonaire est demandée, dépister les fractures vertébrales)

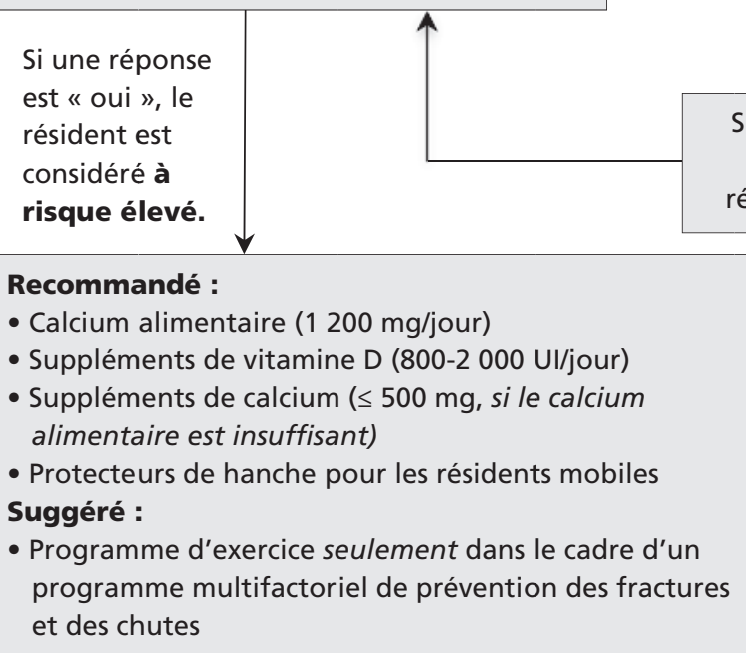

Recommandé :

- Calcium alimentaire (1 $200 \mathrm{mg} / \mathrm{jour}$ )

- Suppléments de vitamine D (800-2 000 Ul/jour)

- Suppléments de calcium ( $\leq 500 \mathrm{mg}$, si le calcium

alimentaire est insuffisant)

- Protecteurs de hanche pour les résidents mobiles

Suggéré :

- Programme d'exercice seulement dans le cadre d'un et des chutes

\section{Stratégies de prévention des fractures et des chutes} (tous les résidents)

Recommandé :

- Calcium alimentaire (1 $200 \mathrm{mg} / \mathrm{jour}$ )

Suggéré :

- Vitamine D ( $\geq 800-2000$ UI/jour)

- Suppléments de calcium ( $\leq 500 \mathrm{mg}$, si le calcium alimentaire est insuffisant)

- Protecteurs de hanche

- Stratégies multifactorielles de prévention des chutes :

1. Exercice (équilibre, force et entraînement fonctionnel)

2. Analyses des médicaments (p. ex., critères de Beers)

3. Évaluation des dangers environnementaux

4. Utilisation d'appareils d'assistance

5. Prise en charge de l'incontinence urinaire

Si le résident subit

une fracture, réévaluer le risque.
Recommandé :

- Alendronate (70 mg par semaine)

- Risedronate (35 mg par semaine ou $150 \mathrm{mg}$ par mois)

- Dénosumab (60 mg par voie sous-cutanée deux fois par année)

- Acide zolédronique (5 mg i.v. une fois par année)

Suggéré :

- Tériparatide (20 $\mu \mathrm{g}$ par voie sous-cutanée chaque jour)

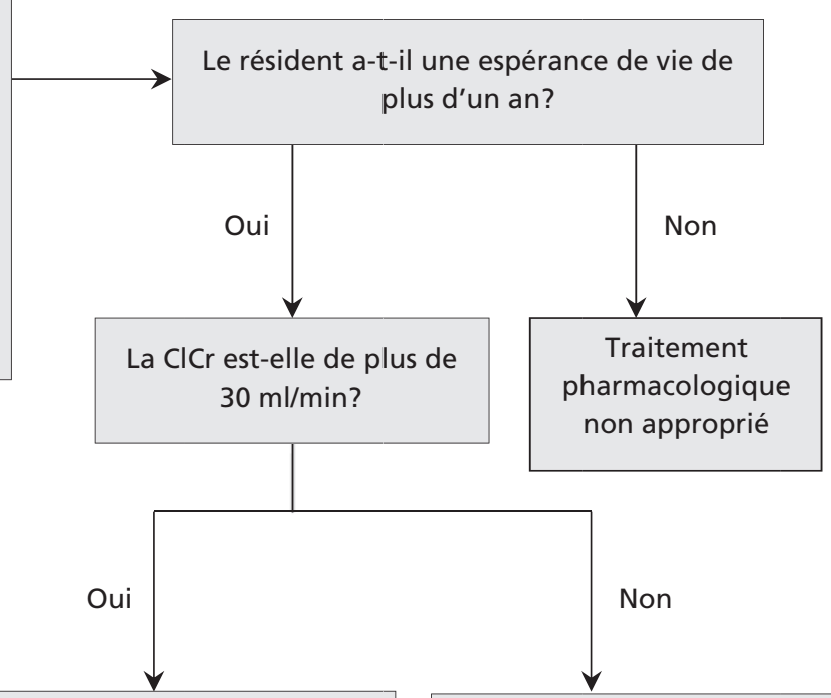

Pour les résidents dont la $\mathrm{ClCr}$ est de 15 à $30 \mathrm{ml} / \mathrm{min}$

Recommandé :

- Dénosumab (60 mg par voie sous-cutanée deux fois par année)

Remarques :

- Surveiller les taux de calcium, étant donné le risque accru d'hypocalcémie

- Les traitements par les bisphosphonates ne sont pas recommandés

- Envisager de diriger le résident vers un spécialiste

Figure 1 : Prévention des fractures pour les résidents d'établissements de soins de longue durée. $\mathrm{ClCr}=\mathrm{clairance}$ de la $\mathrm{créatinine}$, UI = unité internationale, i.v. = par voie intraveineuse. 
tion du risque de fractures de la hanche (10 de moins [IC à $95 \%$ : -14 à 1] pour 1000 sujets). Les données n'étaient pas suffisantes pour explorer les effets des différentes combinaisons d'interventions, ou d'interventions précises, ainsi que leurs coûts humains et financiers.

\section{Mise en œuvre}

Les grandes lignes de la mise en œuvre de ces recommandations se trouvent à la figure 1 . Comme le préconisent les « Guiding Principles for the Care of Older Adults with Multimorbidity » de l'American Geriatrics Society ${ }^{60}$, les préférences des résidents et de leurs aidants doivent être intégrées aux soins. Des outils pour permettre aux responsables d'organisations et aux équipes soignantes d'intégrer les recommandations dans leurs politiques, structures et processus de soins organisationnels sont en cours d'élaboration en partenariat avec les intervenants (Ostéoporose Canada, www.osteoporosecanada.ca; Ontario Osteoporosis Strategy for Long-Term Care, www.osteoporosis longtermcare.ca). Les recommandations seront révisées si de nouvelles options de traitement ou de nouvelles données probantes changent les effets décrits dans cette ligne directrice.

\section{Autres lignes directrices}

Ces recommandations formulées avec l'approche GRADE correspondent aux recommandations pour la prévention de fractures dans les résidences pour aînés de l'Australie ${ }^{61}$ et aux recommandations issues d'un consensus d'experts de l'AMDA - The Society for Post-Acute and Long-Term Care Medicine (anciennement appelée l'American Medical Directors Association $)^{62}$. Cependant, les traitements pour l'ostéoporose offerts aux résidents à risque élevé de fracture varient selon les pays.

La dose optimale de suppléments de vitamine $\mathrm{D}$ pour les aînés a fait l'objet de débats à l'American Geriatrics Society ${ }^{63}$ et à l'US Institute of Medicine ${ }^{64}$. Bien que les deux organisations conviennent qu'une dose de 800 UI entraîne une réduction du nombre de fractures, elles s'entendent moins sur les taux sériques nécessaires à une santé optimale des os et sur la dose nécessaire pour atteindre ce taux chez la majorité des aînés. Comme il est difficile de respecter les exigences de vitamine $\mathrm{D}$ à partir de sources alimentaires et de la lumière du soleil, et compte tenu des données probantes concernant l'innocuité de la vitamine $\mathrm{D}$, Ostéoporose Canada a adopté la position que les personnes à risque élevé de déficience en vitamine D peuvent prendre sans danger un supplément allant jusqu'à 2000 UI par jour ${ }^{65}$.

\section{Lacunes dans les connaissances}

Ces recommandations ont été élaborées après avoir examiné les meilleures données probantes disponibles. Dans la plupart des cas, les données ont été jugées de qualité moyenne ou faible, et de nouvelles recherches pourraient changer l'estimation des effets et notre confiance en cette estimation. En particulier, les données concernant la réduction des fractures obtenue à l'aide de traitements pharmacologiques étaient fondées sur les effets observés chez 100000 femmes postménopausées principalement capables de porter leur poids et courant un risque élevé de fracture, et ont donc été jugées indirectes et déclassées, car elles ne s'appliquent pas directement aux résidents des établissements de soins de longue durée ${ }^{27,38,45}$. Des recherches plus approfondies sont nécessaires de toute urgence pour déterminer l'efficacité et l'innocuité des traitements pour l'ostéoporose lorsqu'ils sont administrés à des résidents ayant une mobilité réduite et des comorbidités multiples. Les initiatives de recherche futures devraient aussi être orientées vers la validation d'un modèle d'évaluation du risque de fracture pour les soins de longue durée ${ }^{66-68}$, l'élaboration de stratégies pour améliorer la consommation alimentaire de calcium, la réalisation d'études sur l'exercice qui mesurent les résultats tels que la douleur, la mobilité, la qualité de vie et les fractures, ainsi que la mise en place d'approches novatrices visant à réduire les blessures causées par les chutes.

\section{Conclusion}

Les objectifs de la prévention des fractures sont de prévenir la douleur, la perte de mobilité, les blessures graves et les transferts vers les soins actifs, et ultimement de maximiser les possibilités de qualité de vie chez les résidents d'établissements de soins de longue durée. Ces lignes directrices donnent des recommandations quant à l'utilisation d'approches aussi bien pharmacologiques que non pharmacologiques pour réduire les fractures en tenant compte des multimorbidités et de l'espérance de vie des résidents.

\section{Références}

1. Papaioannou A, Morin S, Cheuxong AM, et coll. Lignes directrices de pratique clinique 2010 pour le diagnostic et le traitement de l'ostéoporose au Canada : Sommaire. JAMC 2010; 182 : p. 1864-1873.

2. Crilly RG, Tanner DA, Kloseck M, et coll. Hip fractures in long-term care : Is the excess explained by the age and gender distribution of the residents? J Aging Res 2010; 2010 :291258.

3. Ronald LA, McGregor MJ, McGrail KM, et coll. Hospitalization rates of nursing home residents and community-dwelling seniors in British Columbia. Can J Aging 2008; 27 : p. 109-115.

4. Zimmerman S, Chandler JM, Hawkes W, et coll. Effect of fracture on the health care use of nursing home residents. Arch Intern Med 2002; 162 : p. 1502-1508.

5. Nikitovic M, Wodchis WP, Krahn MD, et coll. Direct 
health-care costs attributed to hip fractures among seniors: a matched cohort study. Osteoporos Int 2013; 24 : p. 659-669.

6. Rodondi, A, Chevalley T, Rizzoli R. Prevalence of vertebral fracture on oldest old nursing home residents. Osteoporosis Int 2012; 23 : p. 2601-2606.

7. Papaioannou A, Watts NB, Kendler DL, et coll. Diagnosis and management of vertebral fractures in elderly adults. Am J Med $2002 ; 113$ : p. 220-228.

8. Les soins aux personnes âgées atteintes de la maladie d'Alzheimer et d'autres formes de démence. Ottawa : Institut canadien d'information sur la santé; 2010. Accessible au : https:// secure.cihi.ca/free_products/Dementia_AIB_2010_FR.pdf (consulté le 15 mai 2013).

9. Easterling CS, Robbins E. Dementia and dysphagia. Geriatr Nurs 2008; 29 : p. 275-285.

10. Jackson LD, Little J, Kung E, et coll. Safe medication swallowing in dysphagia: a collaborative improvement project. Healthc $Q 2008$; 11 (3 Spec $\mathrm{N}^{\circ}$ ) : p. 110-116.

11. Papaioannou A, Ray JG, Ferko NC, et coll. Estimation of creatinine clearance in elderly persons in long-term care facilities. Am J Med 2001; 111 : p. 569-573.

12. Garg AX, Papaioannou A, Ferko N, et coll. Estimating the prevalence of renal insufficiency in seniors requiring long-term care. Kidney Int 2004; 65 : p. 649-653.

13. Leslie WD, Berger C, Langsetmo L, et coll. Canadian Multicentre Osteoporosis Study Research Group. Construction and validation of a simplified fracture risk assessment tool for Canadian women and men: results from the CaMos and Manitoba cohorts. Osteoporos Int 2011; 22 : p. 1873-1883.

14. Bravo G, Dubois MF, De Wals P, et coll. Relationship between regulatory status, quality of care, and three-year mortality in Canadian residential care facilities: a longitudinal study. Health Serv Res 2002; 37 : p. 1181-1196.

15. Reid CR. Quality of care and mortality among long-term care residents with dementia. Can Stud Popul 2008; 35 : p. 49-71.

16. Cox L, Kloseck M, Crilly R, et coll. Underrepresentation of individuals 80 years of age and older in chronic disease clinical practice guidelines. Can Fam Physician 2011; 57 : p. 263-269.

17. Mutasingwa DR, Ge H, Upshur RE. How applicable are clinical practice guidelines to elderly patients with comorbidities? Can Fam Physician 2011; 57 : p. 253-262.

18. Guyatt GH, Oxman AD, Vist GE, et coll. GRADE : an emerging consensus on rating quality of evidence and strength of recommendations. BMJ 2008; 336 : p. 924-926.

19. Guyatt GH, Oxman AD, Kunz R, et coll. GRADE Working Group. Going from evidence to recommendations. BMJ 2008; 336 : p. 1049-1051.

20. Kanis JA, Johnell O, Oden A, et coll. The risk and burden of vertebral fractures in Sweden. Osteoporos Int 2004; 15 : p. 20-26.

21. Chandler JM, Zimmerman SI, Girman CJ, et coll. Low bone mineral density and risk of fracture in white female nursing home residents. JAMA 2000; 284 : p. 972-977.

22. Leslie WD, Sadatsafavi M, Lix LM, et coll. Secular decreases in fracture rates 1986-2006 for Manitoba, Canada: a population-based analysis. Osteoporos Int 2011; 22 : p. 2137-2143.

23. Lindsay R, Silverman SL, Cooper C, et coll. Risk of new vertebral fracture in the year following a fracture. JAMA 2001; 285 p. 320-323.

24. Bischoff-Ferrari HA, Willett WC, Orav EJ, et coll. A pooled analysis of vitamin D dose requirements for fracture prevention. N Engl J Med 2012; 367 : p. 40-49.

25. Autier P, Gandini S, Mullie P. A systematic review: influence of vitamin D supplementation on serum 25-hydroxyvitamin D concentration. J Clin Endocrinol Metab 2012; 97 : p. 2606-2613.

26. Avenell A, Gillespie WJ, Gillespie LD, et coll. Vitamin D and vitamin D analogues for preventing fractures associated with involutional and post-menopausal osteoporosis. Base de données Cochrane 2009; (2) : CD000227.

27. Murad MH, Drake MT, Mullan RJ, et coll. Clinical review. Comparative effectiveness of drug treatments to prevent fragility fractures: a systematic review and network meta-analysis. J Clin Endocrinol Metab 2012; 97 : p. 1871-1880.

28. Grant AM, Avenell A, Campbell MK, et coll. RECORD Tria Group. Oral vitamin D3 and calcium for secondary prevention of low-trauma fractures in elderly people (Randomised Evaluation of Calcium or vitamin D, RECORD): a randomised placebocontrolled trial. Lancet 2005; 365 : p. 1621-1628.

29. Muir SW, Montero-Odasso M. Effect of vitamin D supplementation on muscle strength, gait and balance in older adults: a systematic review and meta-analysis. J Am Geriatr Soc 2011; 59 : p. 2291-2300.

30. Murad MH, Elamin KB, Abu Elnour NO, et coll. Clinical review: the effect of vitamin D on falls: a systematic review and meta-analysis. J Clin Endocrinol Metab 2011; 96 : p. 2997-3006.

31. Cameron ID, Gillespie LD, Robertson MC, et coll. Interventions for preventing falls in older people in care facilities and hospitals. Base de données Cochrane 2012; 12 : CD005465.

32. Gillespie LD, Robertson MC, Gillespie WJ, et coll. Intervention for preventing falls in older people living in the community. Base de données Cochrane 2012; 9 : CD007146.

33. Reid IR, Mason B, Horne A, et coll. Randomized controlled trial of calcium in healthy older women. Am J Med 2006; 119 : p. 777-785.

34. Nurmi I, Lüthje P. Incidence and costs of falls and fall injuries among elderly in institutional care. Scand J Prim Health Care 2002; 20 : p. 118-122

35. Elamin MB, Abu Elnour NO, Elamin KB, et coll. Vitamin D and cardiovascular outcomes: a systematic review and meta-analysis. J Clin Endocrinol Metab 2011; 96 : p. 1931-1942.

36. Bolland MJ, Avenell A, Baron JA, et coll. Effect of calcium supplements on risk of myocardial infarction and cardiovascular events: meta-analysis. BMJ 2010; 341 : c3691

37. Bolland MJ, Grey A, Avenell A, et coll. Calcium supplements with or without vitamin D and risk of cardiovascular events: reanalysis of the Women's Health Initiative limited access dataset and meta-analysis. BMJ 2011; 342 : d2040.

38. Levis S, Theodore G. Summary of AHRQ's comparative effectiveness review of treatment to prevent fractures in men and women with low bone density or osteoporosis: update of the 2007 report. J Manag Care Pharm 2012; 18(4 Suppl B) : S1-15.

39. Bischoff-Ferrari HA, Willett WC, Wong JB, et coll. Prevention of nonvertebral fractures with oral vitamin $\mathrm{D}$ and dose dependency: a meta-analysis of randomized controlled trials. Arch Intern Med 2009; 169 : p. 551-561.

40. Fosamax comprimés d'alendronate monosodique [monographie de produit]. Kirkland (QC) : Merck Canada inc.; date de révision : le 4 août 2015. Accessible au : http://www.merck.ca/assets/fr/ pdf/products/FOSAMAX-PM_F.pdf (consulté le 23 août 2015).

41. Actonel comprimés de risedronate sodique [monographie de produit]. Toronto : Warner Chilcott Canada Co.; date de révision : le 15 juillet 2011. Accessible au : www.sanofi.ca/products/ fr/actonel.pdf (consulté le 23 août 2015).

42. Aclasta (injection d'acide zolédronique) [monographie de produit]. Dorval (QC) : Novartis Pharmaceuticals Canada inc.; le 30 juin 2005, date de révision : le 11 septembre 2014. Accessible au : http://www.novartis.ca/fr/products/pharmaceuticals/ index.shtml (consulté le 23 août 2015).

43. Mise à jour sur l'innocuité rénale de Zometa (Acide Zolédronique) et Aclasta (Acide Zolédronique) - Novartis Pharma Canada inc. pour les professionnels de la santé. Ottawa : Santé Canada. Accessible au : http://healthycanadians.gc.ca/recall-alert-rappel-avis/hc-sc/2005/14383a-fra.php (consulté le 23 août 2015).

44. Prolia (denosumab) [monographie de produit]. Mississauga (ON) : Amgen Canada inc.; le 20 octobre 2014. Accessible au : http:// www.amgen.ca/Prolia_PM_Fre.pdf (consulté le 23 août 2015).

45. Hopkins RB, Goeree R, Pullenayegum E, et coll. The relative efficacy of nine osteoporosis medications for reducing the rate of fractures in post-menopausal women. BMC Musculoskelet Disord 2011; 12 : p. 209

46. Bolland MJ, Grey AB, Gamble GD, et coll. Effect of osteoporosis treatment on mortality: a meta-analysis. J Clin Endocrinol Metab 2010; 95 : p. 1174-1181.

47. Nevitt MC, Chen P, Dore RK, et coll. Reduced risk of back pain following teriparatide treatment: a meta-analysis. Osteoporos Int 2006; 17 : p. 273-280

48. Hadji P, Ziller V, Gamerdinger D, et coll. Quality of life and health status with zoledronic acid and generic alendronate - a secondary analysis of the Rapid Onset and Sustained Efficacy (ROSE) study in postmenopausal women with low bone mass. Osteoporos Int 2012; 23 : p. 2043-2051.

49. Sambrook PN, Silverman SL, Cauley JA, et coll. Health-related quality of life and treatment of postmenopausal osteoporosis: results from the HORIZON-PFT. Bone 2011; 48 : p. 1298-1304.

50. Jacobsen DE, Melis RJ, Verhaar HJ, et coll. Raloxifene and tibolone in elderly women: a randomized, double-blind, double-dummy, placebo-controlled trial. J Am Med Dir Assoc 2012; $13:$ e1-7.

51. Silverman S, Viswanathan HN, Yang YC, et coll. Impact of clinical fractures on health-related quality of life is dependent on time of assessment since fracture: results from the FREEDOM trial. Osteoporos Int 2012; 23 : p. 1361-1369.

52. Rizzoli R, Burlet N, Cahall D, et coll. Osteonecrosis of the jaw and bisphosphonate treatment for osteoporosis. Bone 2008; 42 p. 841-847.

53. Rizzoli R, Reginster JY, Boonen S, et coll. Adverse reactions and drug-drug interactions in the management of women with postmenopausal osteoporosis. Calcif Tissue Int 2011; 89 : p. 91-104.

54. Rizzoli R, Åkesson K, Bouxsein M, et coll. Subtrochanteric fractures after long-term treatment with bisphosphonates: a European Society on Clinical and Economic Aspects of Osteoporosis and Osteoarthritis, and International Osteoporosis Foun- 
dation working group report. Osteoporos Int 2011; 22 : p. 373-390.

55. Mak A, Cheung MW, Ho RC, et coll. Bisphosphonates and atrial fibrillation: Bayesian meta-analyses of randomized controlled trials and observational studies. BMC Musculoskelet Disord 2009; 10 : p. 113

56. Grady D, Cauley JA, Stock JL, et coll. Effect of raloxifene on all-cause mortality. Am J Med 2010; 123 : e1-7.

57. Toulis KA, Anastasilakis AD. Increased risk of serious infections in women with osteopenia or osteoporosis treated with denosumab. Osteoporos Int 2010; 21 : p. 1963-1964.

58. Santesso N, Carrasco-Labra A, Brignardello-Petersen R. Hip protectors for preventing hip fractures in older people. Base de données Cochrane 2014; 3 : CD001255.

59. American Geriatrics Society updated Beers criteria for potentially inappropriate medication use in older adults. New York American Geriatrics Society; 2012. Accessible au : http://geriatricscareonline.org/ProductAbstract/american-geriatrics-society-updated-beers-criteria-for-potentially-inappropriate-medication-use-in-older-adults/CL001 (consulté le 20 août 2015).

60. American Geriatrics Society Expert Panel on the Care of Older Adults with Multimorbidity. Guiding principles for the care of older adults with multimorbidity: an approach for clinicians. J Am Geriatr Soc 2012; 60 : E1-25.

61. Duque G, Close JJ, deJager JP, et coll. Treatment for osteoporosis in Australian residential age care facilities. Med J Aust 2010; 193 : p. $173-179$.

62. Osteoporosis and fracture prevention in the long-term care setting: clinical practice guideline. Columbia (MD) : American Medical Directors Association; 2009.

63. American Geriatrics Society Workgroup on Vitamin D. Supplementation for older adults. Recommendations abstracted from the American Geriatrics Society consensus statement on vitamin $\mathrm{D}$ for prevention of falls and their consequences. J Am Geriatr Soc 2014; 62 : p. 147-152

64. Rosen CJ, Abrams SA, Aloia JF, et coll. IOM committee members respond to Endocrine Society vitamin D guideline. J Clin Endocrinol Metab 2012; 97 : p. 1146-1152.

65. Hanley DA, Cranney A, Jones G, et coll. Vitamin D in adult health and disease: a review and guideline statement from Osteoporosis Canada. JAMC 2010; 182 : E610-618.

66. Girman CJ, Chandler JM, Zimmerman SI, et coll. Prediction of fracture in nursing home residents. J Am Geriatr Soc 2002; 50 : p. 1341-1347.

67. Colón-Emeric CS, Biggs DP, Schenck AP, et coll. Risk factors for hip fracture in skilled nursing facilities: Who should be evaluated? Osteoporos Int 2003; 14 : p. 484-489.

68. Chen JS, Sambrook PN, Simpson JM, et coll. A selection strategy was developed for fracture reduction programs in frail older people. J Clin Epidemiol 2010; 63 : p. 679-685.

Intérêts concurrents : Alexandra Papaioannou a reçu des subventions et du soutien à la recherche d'Amgen, d'Eli Lilly, de Merck et de Warner Chilcott, ainsi que des honoraires de conférencière d'Amgen, d'Eli Lilly et de Merck. Suzanne Morin a reçu des subventions de recherche d'Amgen et de Merck, et des frais de consultation d'Amgen et d'Eli Lilly. Jonathan Adachi a participé à des essais cliniques d'Amgen, d'Eli Lilly, de Merck et de Novartis, et a reçu des frais de consultation et des honoraires de conférencier d'Amgen, d'Eli Lilly, de Merck, de Novartis et de Warner Chilcott. Richard Crilly fait partie du Conseil consultatif national d'Amgen. Lora Giangregorio a reçu des subventions et du soutien à la recherche de Merck. Robert Josse a siégé au conseil consultatif et a reçu des honoraires de conférencier et/ ou des subventions de recherche d'Eli Lilly, d'Amgen et de Merck. Hope Weiler a reçu du financement des Producteurs laitiers du Canada. Susan Whiting est conseillère en éducation chez Yoplait France et Pfizer Soins de santé, et a reçu du financement de recherche des Producteurs laitiers du Canada et de la Saskatchewan Health Research Foundation. Angela Cheung a reçu des subventions et des honoraires d'Amgen,
d'Eli Lilly et de Merck. Alexandra Papaioannou, Suzanne Morin, Sidney Feldman, Robert Josse et Angela Cheung sont membres du Comité des lignes directrices du conseil consultatif scientifique d'Ostéoporose Canada. Aucun autre intérêt concurrent n'a été déclaré.

Affiliations : Département de médecine (Papaioannou, Adachi, Pickard), Département d'épidémiologie clinique et de biostatistique (Papaioannou, Santesso) et École des sciences infirmières (Kaasalainen), Faculté des sciences de la santé, Université McMaster, Hamilton (Ontario); Centre d'éducation gériatrique et de recherche scientifique sur le vieillissement (Papaioannou, Pickard, Skidmore), St. Peter's Hospital, Hamilton (Ontario); Département de médecine (Morin), Centre universitaire de santé McGill, Montréal (Québec); Hôpital général de Montréal (Morin), Montréal (Québec); Département de médecine (Feldman, Josse, Moser, Cheung) et Département de physiothérapie (Jaglal), Université de Toronto, Toronto (Ontario); Baycrest Geriatric Health Care System (Feldman, Katz, Moser), Toronto (Ontario); Centre de soins de santé StJoseph (Adachi), Hamilton (Ontario); Division de médecine gériatrique, Département de médecine (Crilly), Université Western Ontario, London (Ontario); Département de kinésiologie (Giangregorio), Université de Waterloo, Waterloo (Ontario); Institut de recherche en services de santé (Jaglal), Toronto (Ontario); Li Ka Shing Knowledge Institute (Josse), hôpital St. Michael's, Toronto (Ontario); École de diététique et de nutrition humaine (Weiler), Université McGill, Montréal (Québec); Département de nutrition et de diététique (Whiting), Université de la Saskatchewan, Saskatoon (Saskatchewan); Toronto General Hospital (Cheung), Toronto (Ontario).

Collaborateurs : Alexandra Papaioannou et Nancy Santesso ont dirigé le groupe de recherche responsable de l'analyse et de la synthèse des données probantes, de la formulation des recommandations et de la rédaction de la première version du texte. Nancy Santesso a supervisé l'application de l'approche GRADE d'élaboration des lignes directrices et organisé le groupe d'experts à ce sujet. Tous les auteurs ont contribué à la discussion sur les questions liées aux soins aux aînés et aux soins de l'ostéoporose, à l'exercice et à la nutrition. Tous les auteurs ont passé en revue les recommandations finales, et tous ont passé en revue et approuvé le texte final et acceptent de se porter garants du travail.

Financement : Cette initiative a été soutenue par l'Ontario Osteoporosis Strategy for Long-Term Care, qui est financée par le ministère de la Santé et des Soins de longue durée de l'Ontario et Ostéoporose Canada. Le soutien des Instituts de recherche en santé du Canada (MAG - 119120) a permis à de nombreux intervenants des soins de longue durée de participer au groupe d'experts sur les lignes directrices et de se rencontrer en personne. Des subventions sans restrictions pour l'éducation ont été fournies par Amgen inc., Eli Lilly Canada inc., Merck Canada inc. et Warner Chilcott.

Remerciements : Les auteurs sont reconnaissants pour la participation et le soutien de nombreux intervenants des milieux des soins de longue durée et de l'ostéoporose : MaryLou van der Horst, D ${ }^{\text {re }}$ Amy Maher, $D^{r}$ Christopher Frank, $D^{\text {re }}$ Angela Juby, D ${ }^{\text {re }}$ Sharon Marr, D ${ }^{r}$ Manuel MonteroOdasso, D ${ }^{\mathrm{r}}$ Denis O'Donnell, D ${ }^{\mathrm{r}}$ Patrick Quail, D ${ }^{\mathrm{re}}$ Diane Villani, Carol Holmes, Linda Dacres, Dee Lender, Milly Radford, Paula Neves, Kathryn Pilkington, Samantha Peck, Ina Ilse, Kerry Grady, Ravi Jain, Lisa Campbell, Monica Menecola, George Ioannidis, $D^{\text {re }}$ Courtney Kennedy, D ${ }^{\text {re }}$ Janet Pritchard, Keya Shah, Osman Osman et Dr Robert Hopkins. 